\title{
High sensitivity of fluorine gas-assisted FIB-TOF-SIMS for chemical characterization of buried sublayers in thin films
}

\author{
Agnieszka Priebe ${ }^{1, *}$, Laszlo Pethö ${ }^{1}$, Emese Huszar ${ }^{1,2}$, Tianle Xie ${ }^{1,3}$, Ivo Utke ${ }^{1}$ and Johann \\ Michler $^{1}$ \\ 'Empa, Swiss Federal Laboratories for Materials Science and Technology, Laboratory for Mechanics of \\ Materials and Nanostructures, Feuerwerkerstrasse 39, $\mathrm{CH}-3602$ Thun, Switzerland \\ ${ }^{2}$ Laboratory for Nanometallurgy, Department of Materials, ETH Zurich, Vladimir-Prelog-Weg 5, 8093, \\ Zurich, Switzerland \\ ${ }^{3}$ College of Material Science and Engineering, Hunan University, 2 Lushan S Rd, Yuelu, Changsha, \\ 410o82, P.R. China
}

*Corresponding author: agnieszka.priebe@empa.ch

\section{TABLE OF CONTENT}

Table S1. PVD deposition parameters of $\mathrm{Al}_{2} \mathrm{O}_{3} / \mathrm{Ni} / \mathrm{Al}_{2} \mathrm{O}_{3} / \mathrm{Au} / \mathrm{Al}_{2} \mathrm{O}_{3} / \mathrm{Cu} / \mathrm{Al}_{2} \mathrm{O}_{3}$

Table S2: ALD deposition parameters of $\mathrm{Al}_{2} \mathrm{O}_{3} / \mathrm{Ni} / \mathrm{Al}_{2} \mathrm{O}_{3} / \mathrm{Au} / \mathrm{Al}_{2} \mathrm{O}_{3} / \mathrm{Cu} / \mathrm{Al}_{2} \mathrm{O}_{3}$

Table $\mathrm{S}_{3}$. PVD deposition parameters of $\mathrm{ZrMoAg} / \mathrm{Al}_{2} \mathrm{O}_{3} / \mathrm{Mo} / \mathrm{Al}_{2} \mathrm{O}_{3} / \mathrm{Ag} / \mathrm{Al}_{2} \mathrm{O}_{3} / \mathrm{Zr}$

Table $\mathrm{S}_{4}$ : ALD deposition parameters of $\mathrm{ZrMoAg} / \mathrm{Al}_{2} \mathrm{O}_{3} / \mathrm{Mo} / \mathrm{Al}_{2} \mathrm{O}_{3} / \mathrm{Ag} / \mathrm{Al}_{2} \mathrm{O}_{3} / \mathrm{Zr}$.

Table $\mathrm{S}_{5}$. PVD deposition parameters of $\mathrm{Al} / \mathrm{Al}_{2} \mathrm{O}_{3} / \ldots / \mathrm{Al} / \mathrm{Al}_{2} \mathrm{O}_{3} /$

Table S6: ALD deposition parameters of $\mathrm{Al} / \mathrm{Al}_{2} \mathrm{O}_{3} / \ldots / \mathrm{Al} / \mathrm{Al}_{2} \mathrm{O}_{3} / \ldots$

Figure $\mathrm{S}$. The SEM images of mechanically cleaved cross-sections

Figure S2. TEM results of $\mathrm{Al} / \mathrm{Al}_{2} \mathrm{O}_{3} / \ldots / \mathrm{Al} / \mathrm{Al}_{2} \mathrm{O}_{3} / \ldots / \mathrm{Al}$

Figure $\mathrm{S}_{3}$. The depth profiles of $\mathrm{Al}_{2} \mathrm{O}_{3} / \mathrm{Ni} / \mathrm{Al}_{2} \mathrm{O}_{3} / \mathrm{Au} / \mathrm{Al}_{2} \mathrm{O}_{3} / \mathrm{Cu} / \mathrm{Al}_{2} \mathrm{O}_{3}$

Figure $\mathrm{S}_{4}$. Normalized to 1 TOF-SIMS depth profiles of $\mathrm{Al}_{2} \mathrm{O}_{3} / \mathrm{Ni} / \mathrm{Al}_{2} \mathrm{O}_{3} / \mathrm{Au} / \mathrm{Al}_{2} \mathrm{O}_{3} / \mathrm{Cu} / \mathrm{Al}_{2} \mathrm{O}_{3}$

Figure $\mathrm{S}_{5}$. TOF-SIMS depth profiles of the $\mathrm{ZrMoAg} / \mathrm{Al}_{2} \mathrm{O}_{3} / \mathrm{Mo} / \mathrm{Al}_{2} \mathrm{O}_{3} / \mathrm{Ag} / \mathrm{Al}_{2} \mathrm{O}_{3} / \mathrm{Zr}$.

Figure S6. The normalized to 1 TOF-SIMS depth profiles of the $\mathrm{ZrMoAg} / \mathrm{Al}_{2} \mathrm{O}_{3} / \mathrm{Mo} / \mathrm{Al}_{2} \mathrm{O}_{3} / \mathrm{Ag} / \mathrm{Al}_{2} \mathrm{O}_{3} / \mathrm{Zr}$

Figure $\mathrm{S}_{7}$. The normalized to 1 TOF-SIMS depth profiles of signals acquired at masses 90, 106 and 107

Figure S8. The normalized to 1 depth profile of the ${ }^{27} \mathrm{Al}+$ signal measured in $\mathrm{Al} / \mathrm{Al}_{2} \mathrm{O}_{3} / \mathrm{Al} / \mathrm{Al}_{2} \mathrm{O}_{3} /$.../Al.

Figure S9. Depth profile of the ${ }^{27} \mathrm{Al}^{+}$signal measured in $\mathrm{Al} / \mathrm{Al}_{2} \mathrm{O}_{3} / \mathrm{Al} / \mathrm{Al}_{2} \mathrm{O}_{3} / \ldots / \mathrm{Al}$ 
Table S1. PVD deposition parameters of $\mathrm{Al}_{2} \mathrm{O}_{3} / \mathrm{Ni} / \mathrm{Al}_{2} \mathrm{O}_{3} / \mathrm{Au} / \mathrm{Al}_{2} \mathrm{O}_{3} / \mathrm{Cu} / \mathrm{Al}_{2} \mathrm{O}_{3}$ multilayer sample.

\begin{tabular}{|c|c|c|c|c|c|c|c|}
\hline Layer & $\begin{array}{l}\text { Power } \\
\text { mode }\end{array}$ & $\begin{array}{l}\text { Plasma power } \\
{[\mathrm{W}]}\end{array}$ & $\begin{array}{l}\text { Plasma current } \\
{[\mathrm{mA}]}\end{array}$ & $\begin{array}{l}\text { Deposition } \\
\text { time }[\mathrm{s}]\end{array}$ & $\begin{array}{l}\text { Base pressure } \\
{[\mathrm{mbar}]}\end{array}$ & $\begin{array}{l}\text { Process } \\
\text { pressure } \\
\text { [mbar] }\end{array}$ & $\begin{array}{l}\text { Argon } \\
\text { flow } \\
{[\mathrm{sccm}]}\end{array}$ \\
\hline $\mathrm{Cu}$ & DC & 90 & 200 & 240 & \multirow{3}{*}{$8 \times 10^{-7}$} & \multirow{3}{*}{$5 \times 10^{-3}$} & \multirow{3}{*}{15} \\
\hline $\mathrm{Au}$ & $\mathrm{DC}$ & 53 & 100 & 360 & & & \\
\hline $\mathrm{Ni}$ & $\mathrm{RF}$ & 150 & N/A & 830 & & & \\
\hline
\end{tabular}

Table S2: $\mathrm{ALD}$ deposition parameters of $\mathrm{Al}_{2} \mathrm{O}_{3} / \mathrm{Ni} / \mathrm{Al}_{2} \mathrm{O}_{3} / \mathrm{Au} / \mathrm{Al}_{2} \mathrm{O}_{3} / \mathrm{Cu} / \mathrm{Al}_{2} \mathrm{O}_{3}$ multilayer sample.

\begin{tabular}{|c|c|c|c|c|c|c|}
\hline \multicolumn{4}{|l|}{ Precursor } & \multirow{3}{*}{$\begin{array}{l}\text { Substrate tem- } \\
\text { perature }\left[{ }^{\circ} \mathrm{C}\right]\end{array}$} & \multirow{3}{*}{$\begin{array}{l}\text { Argon } \\
{[\mathrm{sccm}]}\end{array}$} & \multirow{3}{*}{ flow } \\
\hline \multicolumn{2}{|l|}{$\mathrm{H}_{2} \mathrm{O}$} & \multicolumn{2}{|l|}{$\mathrm{Al}\left(\mathrm{CH}_{3}\right)_{3}$} & & & \\
\hline $\begin{array}{l}\text { Precursor tem- } \\
\text { perature }\left[{ }^{\circ} \mathrm{C}\right]\end{array}$ & Pulse time [ms] & $\begin{array}{l}\text { Precursor tem- } \\
\text { perature }\left[{ }^{\circ} \mathrm{C}\right]\end{array}$ & Pulse time [ms] & & & \\
\hline 100 & 50 & 100 & 50 & 120 & 50 & \\
\hline
\end{tabular}

Table $\mathrm{S}_{3}$. PVD deposition parameters of $\mathrm{ZrMoAg} / \mathrm{Al}_{2} \mathrm{O}_{3} / \mathrm{Mo} / \mathrm{Al}_{2} \mathrm{O}_{3} / \mathrm{Ag} / \mathrm{Al}_{2} \mathrm{O}_{3} / \mathrm{Zr}$ multilayer sample.

\begin{tabular}{|c|c|c|c|c|c|c|c|c|}
\hline \multicolumn{2}{|l|}{ Layer } & $\begin{array}{l}\text { Power } \\
\text { mode }\end{array}$ & $\begin{array}{l}\text { Plasma } \\
\text { power [W] }\end{array}$ & $\begin{array}{l}\text { Plasma cur- } \\
\text { rent }[\mathrm{mA}]\end{array}$ & $\begin{array}{l}\text { Deposition } \\
\text { time }[\mathrm{s}]\end{array}$ & $\begin{array}{l}\text { Base pres- } \\
\text { sure } \\
\text { [mbar] }\end{array}$ & $\begin{array}{l}\text { Process } \\
\text { pressure } \\
\text { [mbar] }\end{array}$ & $\begin{array}{l}\text { Argon } \\
\text { flow } \\
{[\mathrm{sccm}]}\end{array}$ \\
\hline \multicolumn{2}{|l|}{$\mathrm{Zr}$} & & 73 & & 1385 & $1.8 \times 10^{-7}$ & & \\
\hline \multicolumn{2}{|l|}{$\mathrm{Ag}$} & & 104 & 200 & 700 & $2.3 \times 10^{-7}$ & & \\
\hline \multicolumn{2}{|l|}{ Mo } & C & 67 & & 1760 & $4.7 \times 10^{-7}$ & & \\
\hline \multirow{3}{*}{ ZrMoAg } & $\mathrm{Zr}$ & D & 94 & 250 & \multirow{3}{*}{446} & \multirow{3}{*}{$2.4 \times 10^{-7}$} & $5.0 \times 10^{3}$ & 35 \\
\hline & Mo & & 74 & 220 & & & & \\
\hline & $\mathrm{Ag}$ & & 33 & 80 & & & & \\
\hline
\end{tabular}

Table $\mathrm{S}_{4}$ : ALD deposition parameters of $\mathrm{ZrMoAg} / \mathrm{Al}_{2} \mathrm{O}_{3} / \mathrm{Mo} / \mathrm{Al}_{2} \mathrm{O}_{3} / \mathrm{Ag} / \mathrm{Al}_{2} \mathrm{O}_{3} / \mathrm{Zr}$ multilayer sample.

\begin{tabular}{|c|c|c|c|}
\hline \multicolumn{2}{|l|}{ Precursor } & \multirow{3}{*}{$\begin{array}{l}\text { Substrate tem- } \\
\text { perature }\left[{ }^{\circ} \mathrm{C}\right]\end{array}$} & \multirow{3}{*}{$\begin{array}{l}\text { Argon } \\
\text { [sccm }]\end{array}$} \\
\hline $\mathrm{H}_{2} \mathrm{O}$ & $\mathrm{Al}\left(\mathrm{CH}_{3}\right)_{3}$ & & \\
\hline Pulse time [ms] & Pulse time [ms] & & \\
\hline 0.05 & 0.05 & 90 & 50 \\
\hline
\end{tabular}


Table $\mathrm{S}_{5}$. PVD deposition parameters of $\mathrm{Al} / \mathrm{Al}_{2} \mathrm{O}_{3} / \ldots / \mathrm{Al} / \mathrm{Al}_{2} \mathrm{O}_{3} / \ldots$ multilayer sample. The cycle was repeated 11 times.

\begin{tabular}{|l|l|l|l|l|l|l|l|}
\hline Layer & $\begin{array}{l}\text { Power } \\
\text { mode }\end{array}$ & $\begin{array}{l}\text { Plasma power } \\
{[\mathrm{W}]}\end{array}$ & $\begin{array}{l}\text { Plasma current } \\
{[\mathrm{mA}]}\end{array}$ & $\begin{array}{l}\text { Deposition } \\
\text { time }[\mathrm{s}]\end{array}$ & $\begin{array}{l}\text { Base pressure } \\
{[\mathrm{mbar}]}\end{array}$ & $\begin{array}{l}\text { Process } \\
\text { pressure } \\
{[\mathrm{mbar}]}\end{array}$ & $\begin{array}{l}\text { Argon } \\
\text { flow } \\
{[\mathrm{sccm}]}\end{array}$ \\
\hline $\mathrm{Al}$ & $\mathrm{DC}$ & 131 & 300 & 4324 & $4 \times 10^{-8}$ & $4 \times 10^{-3}$ & 13 \\
\hline
\end{tabular}

Table S6: ALD deposition parameters of $\mathrm{Al} / \mathrm{Al}_{2} \mathrm{O}_{3} / \ldots / \mathrm{Al} / \mathrm{Al}_{2} \mathrm{O}_{3} / \ldots$ multilayer sample. One cycle of $\mathrm{H}_{2} \mathrm{O}+\mathrm{Al}\left(\mathrm{CH}_{3}\right)_{3}$ provided an $\mathrm{Al}_{2} \mathrm{O}_{3}$ layer with approximately $1.2 \AA$ thickness meaning that in order to obtain $1 \mathrm{~nm}$ thick ALD layer 8 cycles were needed. Therefore, subsequent layers were deposited within 8 , $16, . . ., 80$ cycles to provide layer thicknesses of $1 \mathrm{~nm}, 2 \mathrm{~nm}, . ., 10 \mathrm{~nm}$, respectively.

\begin{tabular}{|c|c|c|c|c|c|c|}
\hline \multicolumn{4}{|l|}{ Precursor } & \multirow{3}{*}{$\begin{array}{l}\text { Substrate tem- } \\
\text { perature }\left[{ }^{\circ} \mathrm{C}\right]\end{array}$} & \multirow{3}{*}{$\begin{array}{l}\text { Argon } \\
{[\mathrm{sccm}]}\end{array}$} & \multirow{3}{*}{ flow } \\
\hline \multicolumn{2}{|l|}{$\mathrm{H}_{2} \mathrm{O}$} & \multicolumn{2}{|l|}{$\mathrm{Al}\left(\mathrm{CH}_{3}\right)_{3}$} & & & \\
\hline $\begin{array}{l}\text { Precursor tem- } \\
\text { perature }\left[{ }^{\circ} \mathrm{C}\right]\end{array}$ & Pulse time [ms] & $\begin{array}{l}\text { Precursor tem- } \\
\text { perature }\left[{ }^{\circ} \mathrm{C}\right]\end{array}$ & Pulse time [ms] & & & \\
\hline 100 & 50 & 100 & 50 & 120 & 50 & \\
\hline
\end{tabular}

a)

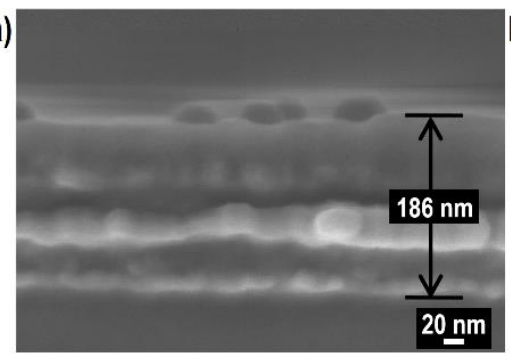

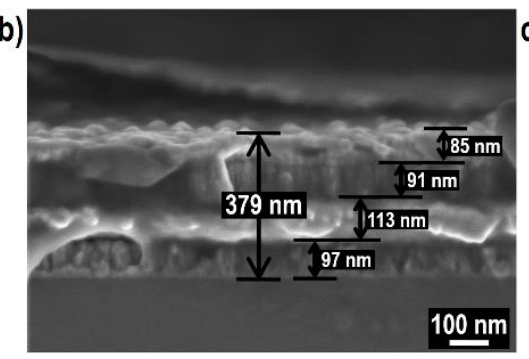

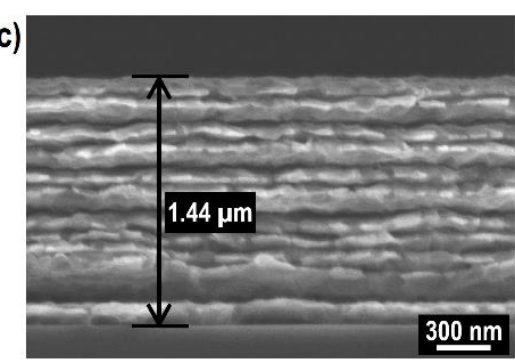

Figure S1. The SEM images of mechanically cleaved cross-sections. Multilayer materials are presented from top to bottom: a) $\mathrm{Al}_{2} \mathrm{O}_{3} / \mathrm{Ni} / \mathrm{Al}_{2} \mathrm{O}_{3} / \mathrm{Au} / \mathrm{Al}_{2} \mathrm{O}_{3} / \mathrm{Cu} / \mathrm{Al}_{2} \mathrm{O}_{3} / \mathrm{Si}$, b) $\mathrm{ZrMoAg} / \mathrm{Al}_{2} \mathrm{O}_{3} / \mathrm{Mo} / \mathrm{Al}_{2} \mathrm{O}_{3} / \mathrm{Ag} / \mathrm{Al}_{2} \mathrm{O}_{3} / \mathrm{Zr} / \mathrm{Si}$ and c) $\mathrm{Al} / \mathrm{Al}_{2} \mathrm{O}_{3} / \mathrm{Al} / \mathrm{Al}_{2} \mathrm{O}_{3} / \ldots / \mathrm{Al} / \mathrm{Si}$.
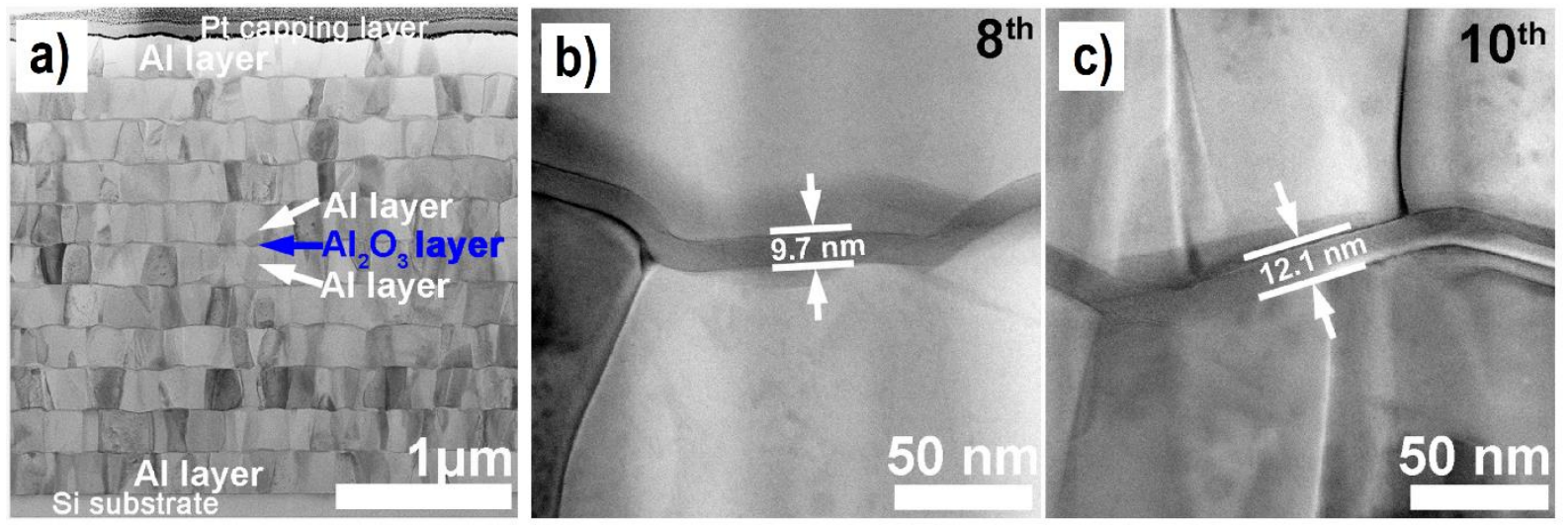

Figure S2. TEM results of $\mathrm{Al} / \mathrm{Al}_{2} \mathrm{O}_{3} / \ldots / \mathrm{Al} / \mathrm{Al}_{2} \mathrm{O}_{3} / \ldots / \mathrm{Al}$ multilayer: a cross-section of TEM lamella (a) and lift-out interfacial images of the $8^{\text {th }}(\mathrm{b})$ and $10^{\text {th }}$ (c) interfaces. Reprinted from Thin Solid Films, Vol. 711, Tianle Xie et. al, "Synthesis of model Al- $\mathrm{Al}_{2} \mathrm{O}_{3}$ multilayer systems with monolayer oxide thickness control by circumventing native oxidation", Pages No. 138287, Copyright (2020), with permission from Elsevier. 

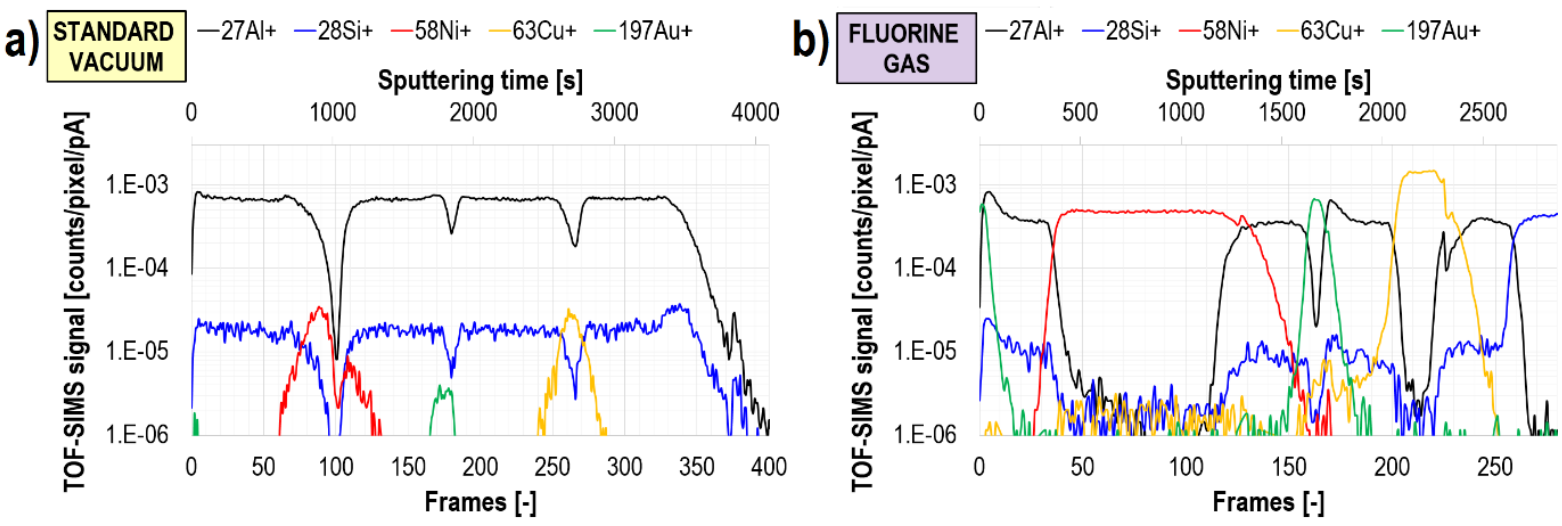

Figure $\mathrm{S}_{3}$. The depth profiles of $\mathrm{Al}_{2} \mathrm{O}_{3} / \mathrm{Ni} / \mathrm{Al}_{2} \mathrm{O}_{3} / \mathrm{Au} / \mathrm{Al}_{2} \mathrm{O}_{3} / \mathrm{Cu} / \mathrm{Al}_{2} \mathrm{O}_{3}$ multilayer sample obtained under standard vacuum conditions (a) and during the fluorine gas-assisted TOF-SIMS measurements (b).
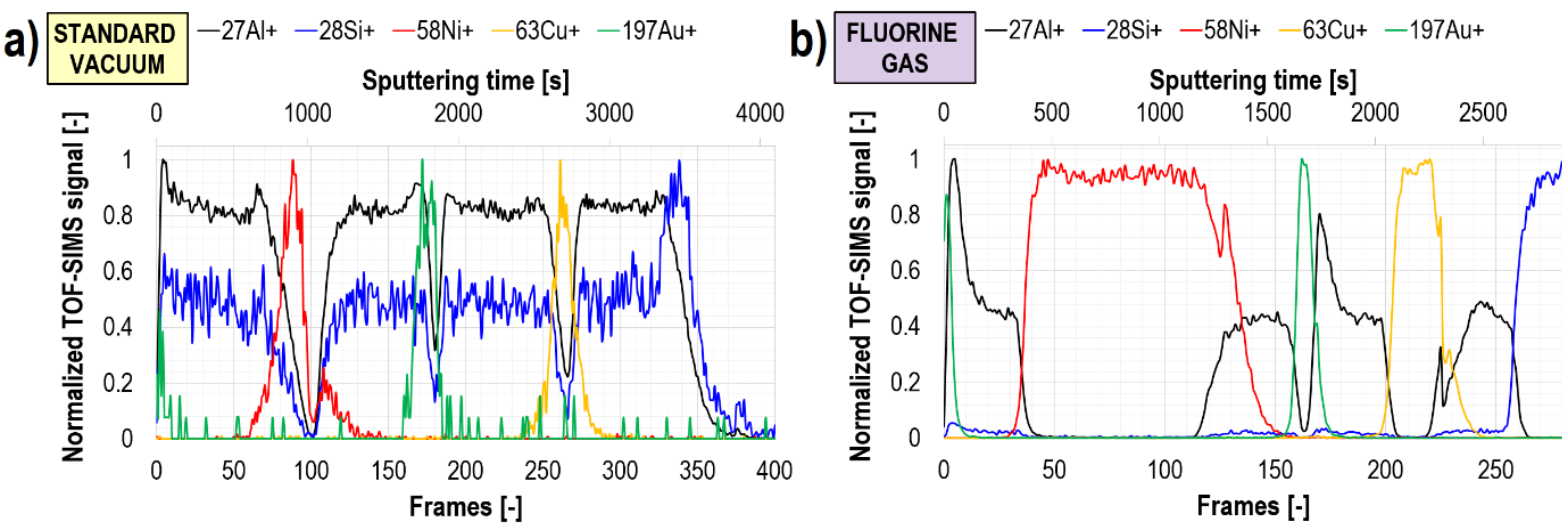

Figure $\mathrm{S}_{4}$. Normalized to 1 TOF-SIMS depth profiles of $\mathrm{Al}_{2} \mathrm{O}_{3} / \mathrm{Ni} / \mathrm{Al}_{2} \mathrm{O}_{3} / \mathrm{Au} / \mathrm{Al}_{2} \mathrm{O}_{3} / \mathrm{Cu} / \mathrm{Al}_{2} \mathrm{O}_{3}$ multilayer acquired under standard vacuum conditions (a) and in the presence of fluorine gas.
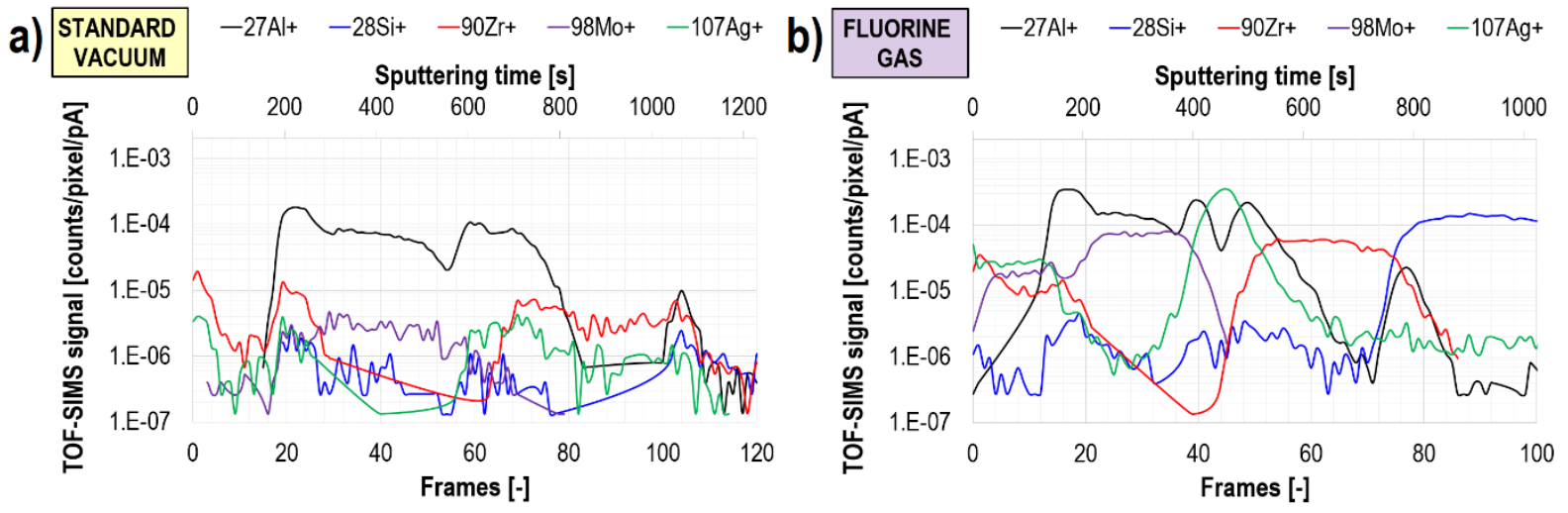

Figure $\mathrm{S}_{5}$. TOF-SIMS depth profiles of the $\mathrm{ZrMoAg} / \mathrm{Al}_{2} \mathrm{O}_{3} / \mathrm{Mo} / \mathrm{Al}_{2} \mathrm{O}_{3} / \mathrm{Ag} / \mathrm{Al}_{2} \mathrm{O}_{3} / \mathrm{Zr}$ multilayer obtained under standard vacuum conditions (a) and with the presence of fluorine gas (b). 
a)

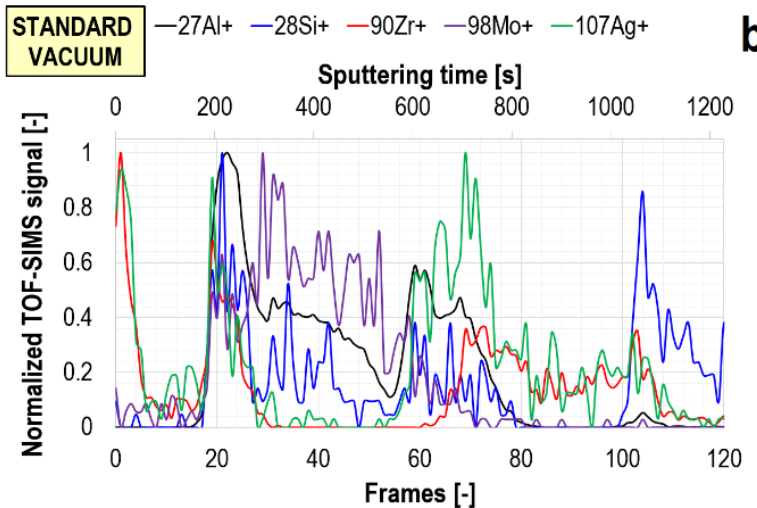

b)

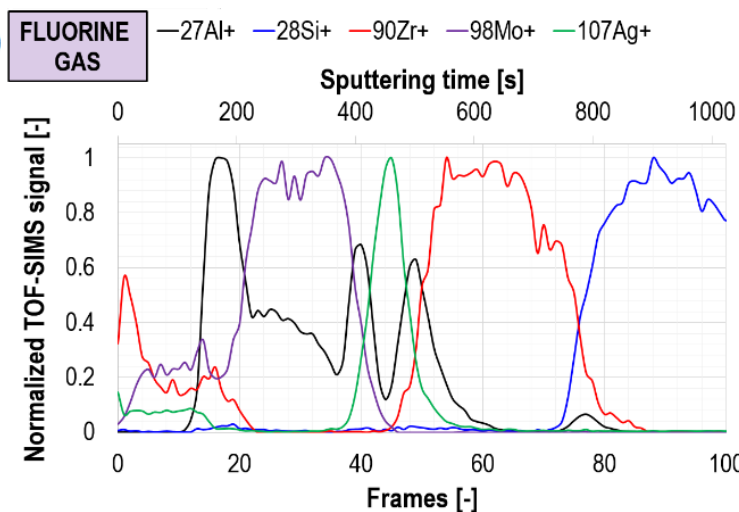

Figure S6. The normalized to 1 TOF-SIMS depth profiles of the $\mathrm{ZrMoAg} / \mathrm{Al}_{2} \mathrm{O}_{3} / \mathrm{Mo} / \mathrm{Al}_{2} \mathrm{O}_{3} / \mathrm{Ag} / \mathrm{Al}_{2} \mathrm{O}_{3} / \mathrm{Zr}$ sample. The data were acquired without (a) and with (b) the presence of supplementary gas. The exact signal values are provided in Figure 5 .

a)

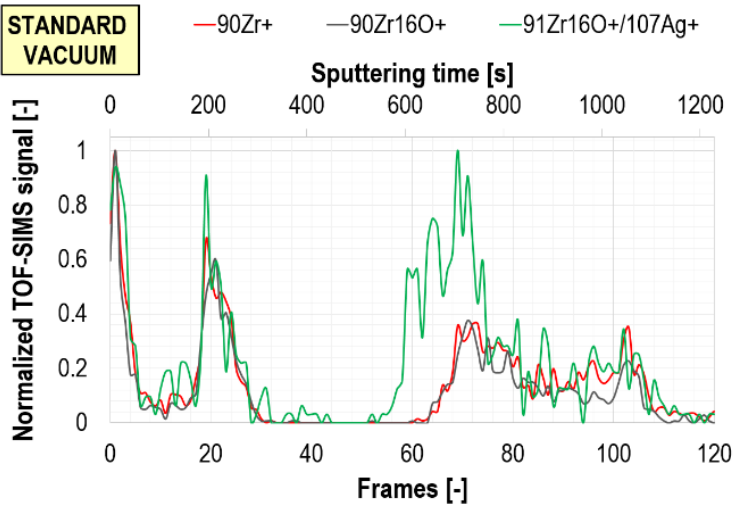

b)

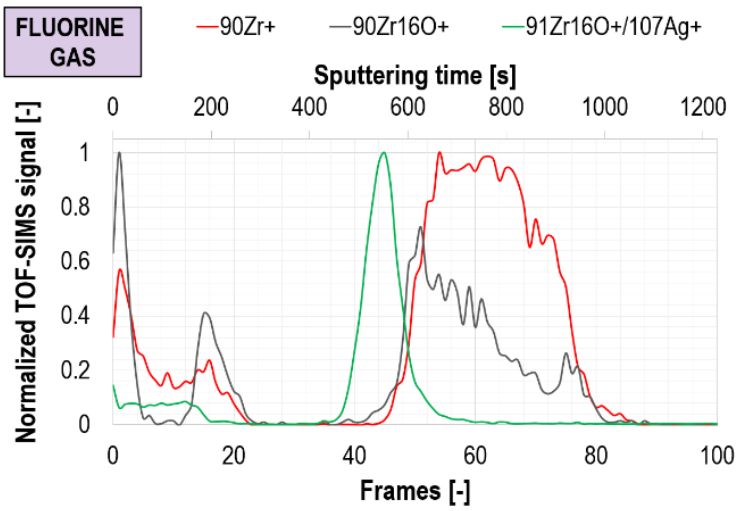

Figure $\mathrm{S}_{7}$. The normalized to 1 TOF-SIMS depth profiles of signals acquired at masses 90, 106 and 107 (corresponding to ${ }^{\circ} \mathrm{Zr}^{+}$signal, ${ }^{90} \mathrm{Zr}^{16} \mathrm{O}^{+}$signal as well as ${ }^{9} \mathrm{Zr}^{16} \mathrm{O}^{+}$and $/$or ${ }^{107} \mathrm{Ag}^{+}$signals): $\mathrm{ZrMoAg} / \mathrm{Al}_{2} \mathrm{O}_{3} / \mathrm{Mo} / \mathrm{Al}_{2} \mathrm{O}_{3} / \mathrm{Ag} / \mathrm{Al}_{2} \mathrm{O}_{3} / \mathrm{Zr}$ sample. The data were obtained without (a) and with (b) the presence of supplementary gas.
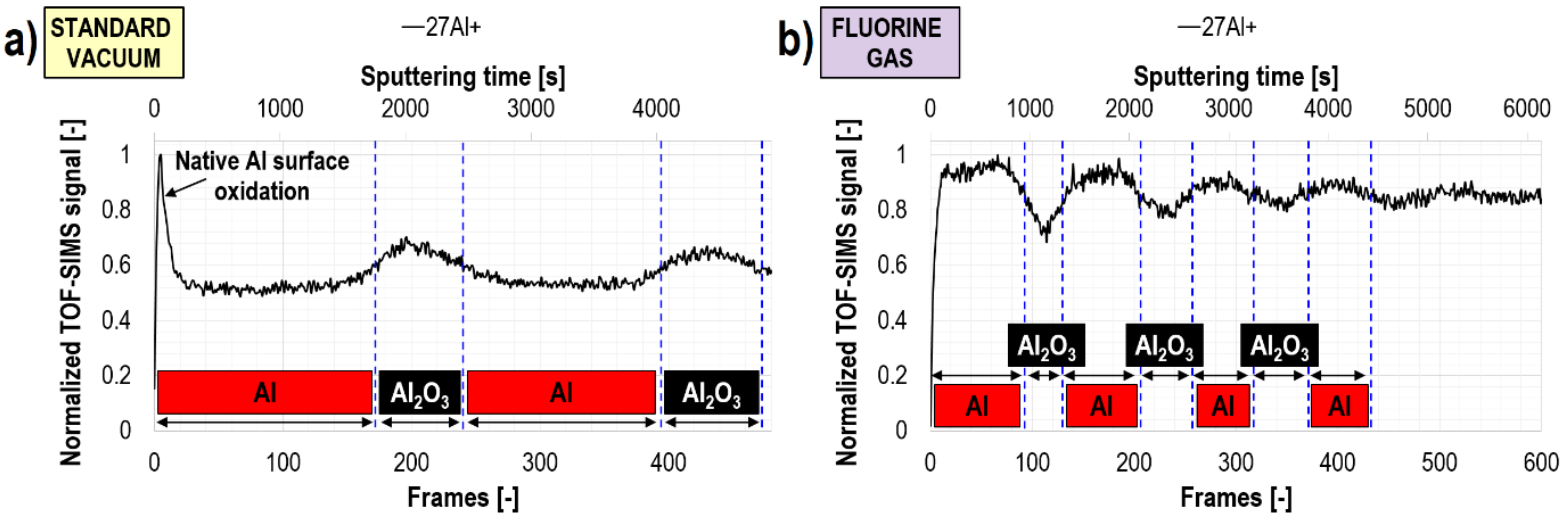

Figure S8. The normalized to 1 depth profile of the ${ }^{27} \mathrm{Al}^{+}$secondary ion signal measured in $\mathrm{Al} / \mathrm{Al}_{2} \mathrm{O}_{3} / \mathrm{Al} / \mathrm{Al}_{2} \mathrm{O}_{3} / \ldots / \mathrm{Al}$ multilayer. The TOF-SIMS data acquired at standard vacuum conditions (a) and in the presence of fluorine gas (b). The blue dashed lines roughly indicate the location of the interfaces (estimated at a half of ascending/descending signal slopes). The absolute signal values are given in Figure S9. 


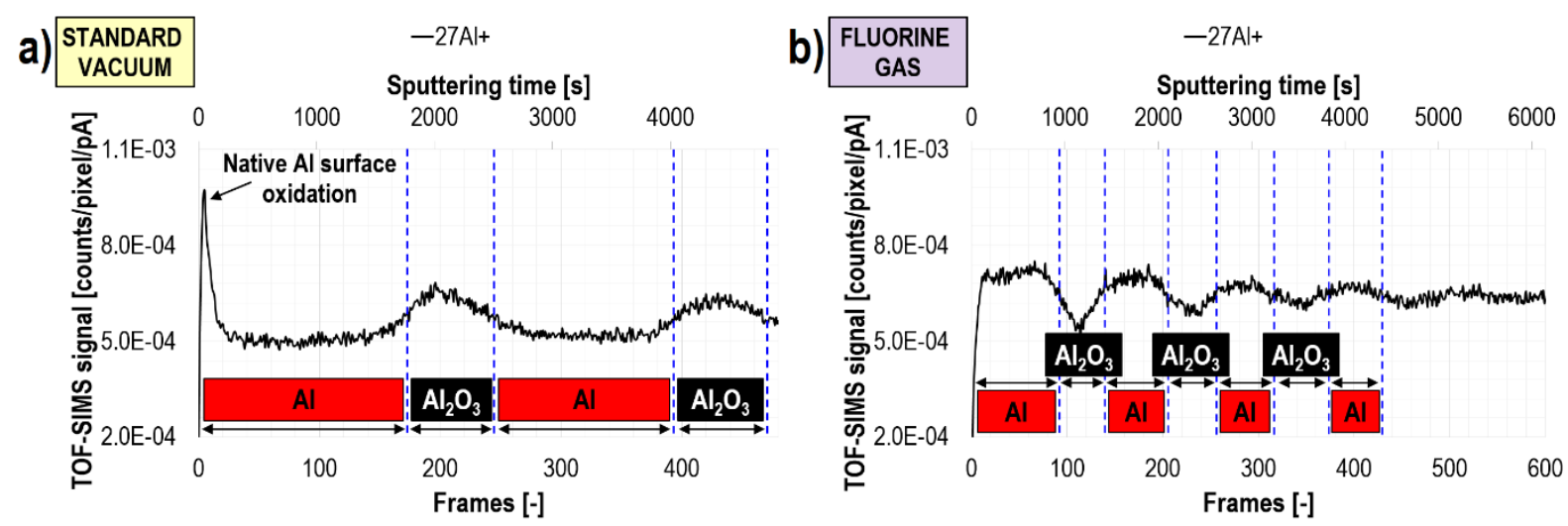

Figure 9 . The depth profile of $\mathrm{Al} / \mathrm{Al}_{2} \mathrm{O}_{3} / \ldots / \mathrm{Al} / \mathrm{Al}_{2} \mathrm{O}_{3} / \ldots / \mathrm{Al}$ multilayer sample acquired using TOF-SIMS under standard vacuum conditions (a) and in the presence of fluorine gas (b). 